\title{
A kind of Dual Mixed Filter Algorithm based on Adaptive Filter and Morphological Filter
}

\author{
Z.C. $\mathrm{Li}$ \\ School of Electrical and Automatic Engineering, Changshu Institute of Technology \\ Changshu, China
}

\begin{abstract}
This paper put forward a new algorithm in response to the extraction of the fundamental component of harmonic current detection. Firstly, principle of harmonic detection was introduced through current detection scheme, and then adaptive filter and morphological filter which can be used as low pass filter were introduced together with their combination. It is proved the algorithm that use adaptive filter to pre-filter the signal and combine morphological filter with it to filter again is better than what of only using adaptive filter or morphological filter within half power frequency period of time, which is better than another form of combination, making the integrated effect of the two algorithms be better.
\end{abstract} filter

Keywords-current detection; adaptive filter; morphological

\section{INTRODUCTION}

The development of power electronic technology in recent years is very rapid, and it is widely used to enhance the production and living standards. However, as the nonlinear load in power distribution system, power electronic devices produce large amounts of harmonics and reactive currents. These cause power quality problems, such as voltage and current distortion, and voltage flicker, and so on. In order to reduce the pollution of power grid, the use of active power filter compensating harmonics and reactive currents[1], has become the focus of research and application fields. According to harmonic current and reactive current component in the power distribution system, active power filter generates the compensation current, which has the same amplitude and opposite polarity, so that offset each other. Only real-time and accurate detection of harmonic currents and reactive current components, power filters can play an effective role in compensating.

At present, the detection method of harmonic current and reactive component widely used is synchronous reference frame (SRF) method, which is developed on the basis of instantaneous reactive power (IRP) theory[2,3]. In the SRF Method framework[4,5], the positive sequence fundamental component of load current is separated out in real time, through the low pass filter (LPF). It is then subtracted from the load current, and harmonic and reactive component can be obtained. The LPF design is the key link, which has become the bottleneck of SRF Method playing advantage in the current detection. Traditional analog filters exist amplitude and phase errors, slow dynamic response[6].Therefore, in recent years, using mathematical morphology algorithm for low-pass filtering[7], or adaptive algorithm to complete the low-pass filtering[8], both of which achieved good results.

In this paper, a novel strategy is comprehensive application of adaptive algorithms and mathematical morphology algorithm to construct a composite low-pass filter It can play integrated effect of the two algorithms to further enhance current detection accuracy. Based on dq theory, current detection scheme is described in Section 2, and the effect of low pass filtering on current detection is further introduced in active power filter. In Section 3, morphological filter is reviewed. Section 4 shows adaptive filter. The composite filter is formulated in Section 5, which is equipped with the pre filter using adaptive algorithm and the main filter using morphological algorithm. Section 6 reports the simulation results of the afore-mentioned algorithms that alone are used as filter. In this section, the performance is also discussed when using them in current detection. Finally, some conclusions are given in Section 7.

\section{CURRENT DETECTION SCHEME}

In order to detect harmonic current instantaneously, signal processing process, as shown in figure1[9]. First of all, transform these three-phased currents ia,ib,ic, with harmonics, into two-phased currents $i \alpha$ and $i \beta$, by the alternation abc/ $\alpha \beta$. And then change it into instantaneous active current ip and instantaneous reactive current iq, throughout the alternation of $\alpha \beta / \mathrm{dq}$. Secondly, the current ip and iq, through the LPF low-pass filter, the DC component can be educed. After two inverse transformations, $\mathrm{dq} / \alpha \beta$ and $\alpha \beta / \mathrm{abc}$, the fundamental component in three-phase currents iaf, ibf, icf can be taken, at this time, these three-phased currents are what have been eliminated the harmonics.

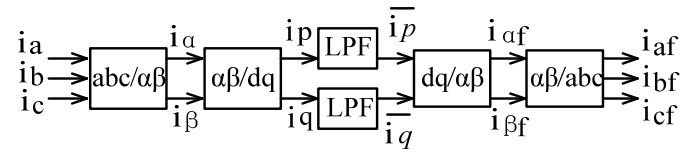

\section{FIGURE I. THE SCHEMATIC OF FUNDAMENTAL SIGNAL DETECTION}

The equations of three-phased currents with harmonics are showed in followed. 


$$
\left\{\begin{array}{l}
i_{a}=\sqrt{2} \sum_{n=1}^{\infty} I_{n} \sin \left(n \omega t+\varphi_{n}\right) \\
i_{b}=\sqrt{2} \sum_{n=1}^{\infty} I_{n} \sin \left(n \omega t+\varphi_{n}-120^{\circ}\right) \\
i_{c}=\sqrt{2} \sum_{n=1}^{\infty} I_{n} \sin \left(n \omega t+\varphi_{n}+120^{\circ}\right)
\end{array}\right.
$$

By using the coordinate transformation matrix abc/ $\alpha \beta$ as shown in the following equation, the instantaneous three-phase current value transformation to $\alpha-\beta$ plane In three-phase circuits. By the transformation of the equation (3), the values of the two-phased transient currents i $\alpha$, i $\beta$ (axis $\alpha$ are perpendicular to axis $\beta$ ) can be received.

$$
\begin{array}{r}
A_{1}=\sqrt{2 / 3}\left[\begin{array}{ccc}
1 & -\frac{1}{2} & -\frac{1}{2} \\
0 & \frac{\sqrt{3}}{2} & -\frac{\sqrt{3}}{2}
\end{array}\right] \\
{\left[\begin{array}{l}
i_{\alpha} \\
i_{\beta}
\end{array}\right]=\mathrm{A}_{1}\left[\begin{array}{l}
i_{a} \\
i_{b} \\
i_{c}
\end{array}\right]=\sqrt{2 / 3}\left[\begin{array}{ccc}
1 & -\frac{1}{2} & -\frac{1}{2} \\
0 & \frac{\sqrt{3}}{2} & -\frac{\sqrt{3}}{2}
\end{array}\right]\left[\begin{array}{l}
i_{a} \\
i_{b} \\
i_{c}
\end{array}\right]=\left[\begin{array}{l}
\sqrt{3} \sum_{n=1}^{\infty} I_{n} \sin \left(n \omega t+\varphi_{n}\right) \\
\sqrt{3} \sum_{n=1}^{\infty}-I_{n} \cos \left(n \omega t+\varphi_{n}\right)
\end{array}\right]}
\end{array}
$$

The following equation (4) is an $\alpha \beta / \mathrm{dq}$ transformation matrix. By using equation (5), currents iaand i $\beta$ in coordinate system $\alpha-\beta$ are changed to coordinate system $d-q$ and the instantaneous active current ip and instantaneous reactive current iq can be received, wherein is seen the fundamental current transform to the d-q coordinate into direct form, other components are alternating forms, facilitating the low-pass filter to extract the fundamental current.

$$
\begin{gathered}
B=\left[\begin{array}{ll}
\sin \omega t & -\cos \omega t \\
-\cos \omega t & -\sin \omega t
\end{array}\right] \\
{\left[\begin{array}{l}
i_{p} \\
i_{q}
\end{array}\right]=B\left[\begin{array}{l}
i_{\alpha} \\
i_{\beta}
\end{array}\right]=\left[\begin{array}{ll}
\sin \omega t & -\cos \omega t \\
-\cos \omega t & -\sin \omega t
\end{array}\right]\left[\begin{array}{l}
i_{\alpha} \\
i_{\beta}
\end{array}\right]=\left[\begin{array}{l}
\sqrt{3} \sum_{n=1}^{\infty} I_{n} \cos \left[(n-1) \omega t+\varphi_{n}\right] \\
\sqrt{3} \sum_{n=1}^{\infty}-I_{n} \sin \left[(n-1) \omega t+\varphi_{n}\right]
\end{array}\right]}
\end{gathered}
$$

The inverse transformation of $\alpha \beta / \mathrm{dq}$ is transformation $\mathrm{dq}$ $/ \alpha \beta$ what transformation $\alpha \beta /$ abc is the inverse transformation of $a b c / \alpha \beta$.

\section{MORPHOLOGICAL FILTER}

Mathematical morphology were found in 1960s by two French mathematician Serra J and Matheron, which takes advantage of its structural elements to match the characteristics of the signal in part, while preserving the main features of signals, to extract the useful information, and to achieve the purpose of noise suppression. Mathematical morphology operation is the expansion and erosion operation as the foundation, then extends a cascade combination of opening and closing operations two concepts and open and close operation based on dilation and erosion operation[10].

Suppose the definition of incoming signal $f(n)$ is a one-dimensional original signal $\{0,1,2, \ldots \ldots, \mathrm{N}-1\}$, and the definition of $g(n)$ is the structural elements of $\{0,1,2, \ldots \ldots$, $\mathrm{M}-1\}$. $(\mathrm{N} \geq \mathrm{M})$. Define $\oplus$ and $\Theta$ as the operational symbol of dilation and erosion, in this way dilation and erosion can be calculated by the following equations.

$$
\begin{aligned}
& \left(\mathrm{f}^{\oplus} \mathrm{g}\right)=\max \{\mathrm{f}(\mathrm{n}-\mathrm{m})+\mathrm{g}(\mathrm{m})\} \\
& \left(\mathrm{f}^{\Theta} \mathrm{g}\right)=\min \{\mathrm{f}(\mathrm{n}+\mathrm{m})-\mathrm{g}(\mathrm{m})\}
\end{aligned}
$$

It is seen through the equation (6) and (7) that the dilation and erosion operation only needs a simple add, subtract, and take the maximum and minimum value. Therefore, the speed of real-time signal processing is faster, and relatively the time delay is sure to be smaller. Through the cascade operation both can get two kinds of morphological operation on the calculation of dilation and erosion operation, namely open and close operation which can be defined in the following equations.

$$
\begin{aligned}
& \left(\mathrm{f}^{\circ} \mathrm{g}\right)=\left(\mathrm{f}^{\Theta} \mathrm{g} \oplus \mathrm{g}\right)(\mathrm{n}) \\
& \left(\mathrm{f}^{\bullet} \mathrm{g}\right)=\left(\mathrm{f}^{\oplus} \mathrm{g} \Theta_{\mathrm{g}}\right)(\mathrm{n})
\end{aligned}
$$

Equation (8) is an open operation that can inhibit the positive impulse noise and enhance negative pulse noise, while equation (9) is a close operation which inhibit the negative impulse noise and enhance positive pulse noise. Morphological filter is a new nonlinear mathematical morphology developed from the filter, as it has the characteristics of parallel and rapid implementation, which has been used in the field of signal processing and image analysis. Considering the positive and negative pulse noise suppression effects, with different length of structural elements, can construct a generalized alternating hybrid filter as follows, making the calculation result more accurate[11].

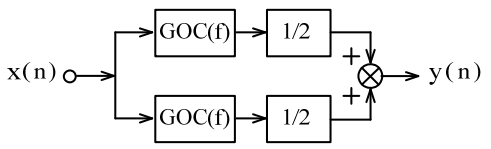

FIGURE II. SCHEMATIC OF GENERALIZED ALTERNATING HYBRID FILTER

The definition of generalized alternating hybrid filter is demonstrated in the following equations, as g1and g2 be structural elements $(\mathrm{g} 1 \subseteq \mathrm{g} 2)$.

$$
\left\{\begin{array}{l}
{[G A H(f)](n)=[G O C(f)+G C O(f)](n) / 2} \\
{[G O C(f)](n)=\left(f \circ g_{1} \bullet g_{2}\right)(n)} \\
{[G C O(f)](n)=\left(f \bullet g_{1} \circ g_{2}\right)(n)}
\end{array}\right.
$$




\section{ADAPTIVE FILTER}

Adaptive filter without prior knowledge of the input signal, according to the characteristics of automatic adjustment of the parameters of digital filter[12]. in order to achieve the optimum filtering. Used as a low pass filter, the basic signal processing as shown in figure 3.

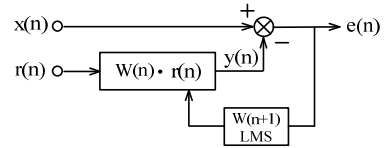

\section{FIGURE III. SCHEMATIC OF ADAPTIVE FILTER}

In the figure above, define $\mathrm{x}(\mathrm{n})$ as input signal, $\mathrm{r}(\mathrm{n})$ as reference signal, $y(n)$ as output signal, $e(n)$ as error feedback signal. Reference signal $r(n)$ generates output signal $y(n)$ through adjustable parameters of digital filter, and compare $y(n)$ with input signal $x(n)$ and get error feedback signal $e(n)$, according to LMS algorithms, taking advantage of error feedback signal $\mathrm{e}(\mathrm{n})$ to adjust the parameter of digital filter, $\mathrm{w}(\mathrm{n}+1)$, and finally approach to optimal filtering performance, minimizing the output error feedback signal e(n).

$$
\left\{\begin{array}{l}
y(n)=w(n) \cdot r(n) \\
e(n)=x(n)-y(n) \\
w(n+1)=w(n)+2 \mu \cdot e(n) \cdot r(n)
\end{array}\right.
$$

Set $\mu$ as factor of step length. According to figure 1, adaptive filter is used as LPF, to extract direct component of ip and iq, set reference signal $\mathrm{r}(\mathrm{n})$ as $\mathrm{DC}$ value 1 . LMS algorithm reduced calculate complex and it is easy to realize, which is suitable for digital filtering.

\section{COMPOSITE FILTER}

In the process of the three-phase fundamental current detection, the core is the designing of LPF low pass filter. Morphological filter and adaptive filter can be used as a low pass filter, and have achieved good results. Cascading the two kinds of filters, one is responsible for signal preprocessing the other is for the main filter, can further enhance the filtering effect. One kind of combination in series is in the form of MAF, namely morphological filter combine with adaptive filter. Use adaptive filter to filter current signal firstly, it is difficult for adaptive filter to make great improvement in filtering because the steady-state precision of morphological filter is higher than what of adaptive filter in response to high speed case.

AMF is an effective combination in series, namely adaptive filter combine with morphological filter, the concept of complex filter is demonstrated in the following sketch. Use adaptive filter to filter current signal primarily, in order to pre-treat signal and ensure its quick reaction. And then, use morphological filter to filter for the second time in order to reach better steady-state precision and to get obviously better complex filtering effects.

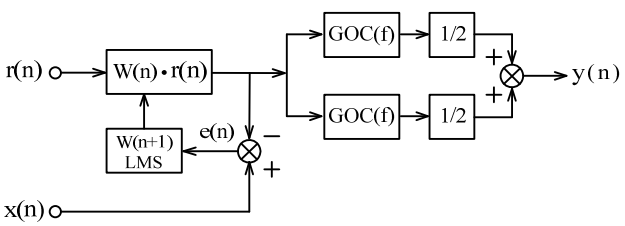

FIGURE IV. SCHEMATIC OF COMPLEX FILTER

In the figure above, signal which is ready to be filtered is set as $x(n)$, and $r(n)$ means reference signal which is equal to 1 , the output signal of LPF is $y(n)$. In adaptive filter, the value of step length factor $\mu$ distinguished between quick reaction and the balance of steady-state. A smaller $\mu$ cause smaller steady-state imbalance although its convergence speed is slow while a bigger $\mu$ can make sure its speed is fast but increase its steady-state imbalance.

\section{SIMULATION ANALYSIS}

This article use Matlab to simulate the algorithm of fundamental current component detection. Analyzes the current with harmonic components in order to verify the effectiveness of the proposed method, these three-phase currents $\mathrm{A}, \mathrm{B}$ and $\mathrm{C}$ are calculated by the following equations containing 5,7and11 times harmonics, with $2 \%$ noise added. The frequency of fundamental wave $\mathrm{f}=50 \mathrm{HZ}$, and the frequency of sampling fs $=1000 \mathrm{HZ}$, after take samples of 1600 points namely 8 periods.

$$
\begin{aligned}
& i_{a}=\sin a t-\frac{1}{5} \sin \left(5 a+\frac{\pi}{6}\right)-\frac{1}{7} \sin \left(7 a t+\frac{\pi}{2}\right)+\frac{1}{11} \sin \left(11 a t+\frac{\pi}{4}\right)+\frac{1}{13} \sin \left(13 a t+\frac{\pi}{3}\right) \\
& i_{b}=\sin \left(a t-\frac{2 \pi}{3}\right)-\frac{1}{5} \sin \left(5 a t+\frac{\pi}{6}-5 * \frac{2 \pi}{3}\right)-\frac{1}{7} \sin \left(7 a t+\frac{\pi}{2}-7 * \frac{2 \pi}{3}\right)+\frac{1}{11} \sin \left(11 a t+\frac{\pi}{4}-11 * \frac{2 \pi}{3}\right)+\frac{1}{13} \sin \left(13 a t+\frac{\pi}{3}-13 * \frac{2 \pi}{3}\right) \\
& i_{c}=\sin \left(a t+\frac{2 \pi}{3}\right)-\frac{1}{5} \sin \left(5 a t+\frac{\pi}{6}+5 * \frac{2 \pi}{3}\right)-\frac{1}{7} \sin \left(7 a b+\frac{\pi}{2}+7 * \frac{2 \pi}{3}\right)+\frac{1}{11} \sin \left(11 \omega t+\frac{\pi}{4}+11 * \frac{2 \pi}{3}\right)+\frac{1}{13} \sin \left(13 a b+\frac{\pi}{3}+13 * \frac{2 \pi}{3}\right)
\end{aligned}
$$

For the advantages of the new algorithm, LPF takes the forms of MF (Morphological filter), AF (Adaptive filter), MAF and AMF in turn. The algorithm of mathematical morphology uses the flat structure elements, set $\mathrm{m}$ as width and $\mu$ as the step length factor of adaptive filter.

TABLE I. COMPARISONS OF THE ALGORITHM

\begin{tabular}{ccccc}
\hline $\begin{array}{c}\text { filter } \\
\text { parameters }\end{array}$ & $\begin{array}{c}\text { filter } \\
\text { form }\end{array}$ & $\begin{array}{c}\text { A phase } \\
\text { MSE }\end{array}$ & $\begin{array}{c}\text { B phase } \\
\text { MSE }\end{array}$ & $\begin{array}{c}\text { C phase } \\
\text { MSE }\end{array}$ \\
\hline $\mathrm{m}=17$ & MF & 0.0266 & 0.0344 & 0.0266 \\
\hline \multirow{2}{*}{$=0.05$} & AF & 0.0884 & 0.0882 & 0.0884 \\
& MAF & 0.0604 & 0.0618 & 0.0604 \\
& AMF & 0.0255 & 0.0284 & 0.0255 \\
\hline \multirow{2}{*}{$=0.04$} & AF & 0.0734 & 0.0732 & 0.0734 \\
$\mathrm{~m}=17$ & MAF & 0.0610 & 0.0613 & 0.0610 \\
& AMF & 0.0240 & 0.0267 & 0.0240 \\
\hline$=0.03$ & AF & 0.0568 & 0.0567 & 0.0568 \\
$\mathrm{~m}=17$ & MAF & 0.0607 & 0.0618 & 0.0607 \\
& AMF & 0.0226 & 0.0255 & 0.0226 \\
\hline
\end{tabular}

The algorithm what it is shown in table 1 have common prerequisite of less than $10 \mathrm{~ms}$ of response time. The following chart further demonstrates the comparison of MAF and AMF. 


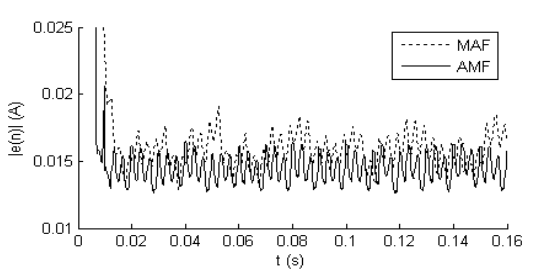

FIGURE V. EFFECT PICTURE OF COMBINATION OF MORPHOLOGICAL FILTER AND ADAPTIVE

The algorithm AMF has better precision according to the chart above. Simulation of using the algorithm to extract fundamental component of three-phase current are shown in the chart below. The transition time of filtering process is spent for half a fundamental cycle.

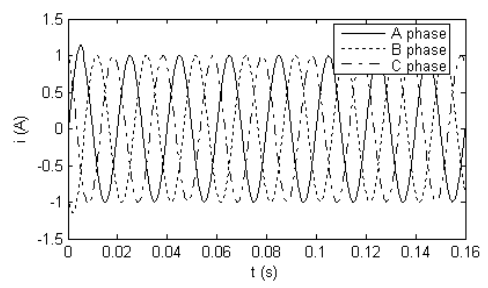

FIGURE VI. SIMULATION OF EXTRACT FUNDAMENTAL COMPONENT OF THREE-PHASE CURRENT AFTER FILTERING

\section{CONCLUSION}

This parchment put forward a kind of dual mixed filter algorithm based on adaptive filter and morphological filter which take the signal preprocessing method of using adaptive filter to pre-filter the signal and combining morphological filter with it to filter again. The filtering property of this algorithm is better than what of only using adaptive filter or morphological filter or MAF in condition of half power frequency cycle time limit. The algorithm is simple, fast and practical, morphological filter there is some space for improvements in the condition of adaptive filter using an alterable $\mu$ and morphological filter selecting a more reasonable length.

\section{REFERENCES}

[1] JuntaoFei,KaiqiMa,ShengleiZhang,et al. Adaptive CurrentControlwith PI-FuzzyCompound Controller for ShuntActive Power Filter. Mathematical Problems in Engineering, vol. 2013, pp. 1-11,2013.

[2] H. Akagi, Y. Kanazawa, and A. Nabae, Instantaneous reactive power compensators comprising switching devices without energy storage components, IEEE Trans. Ind. Applicat., 20(3), pp. 625-630, 1984.

[3] R. S. Herrera, P. Salmeron, and H. Kim, Instantaneous reactive power theory applied to active power filter compensation: Different approaches, assessment, and experimental results, IEEE Trans. Ind. Electron, 55(1), pp. 184-196,2008.

[4] Soares V, Verdelho P, Marques G, An instantaneous active and reactive current component method for active filters, IEEE Trans Power Electron, $15(4)$, pp. 660-669,2000.

[5] B. Singh and V. Verma, Selective compensation of power quality problems through active power filter by current decomposition, IEEE Trans. Power Del. 23(2), pp. 792-799,2008.

[6] Hongyu Li, Fang Zhuo, Zhaoan Wang,et al, A Novel Time-Domain Current-Detection Algorithm for Shunt Active Power Filters,IEEE Transactions on Power Systems, 20(2), pp. 644-651,2005.
[7] Z. Lu, Q. H. Wu,J. Fitch,A Morphological Filter For Estimation of Power System Harmonics,2006 International Conference on Power System Technology, IEEE: Chongqing, pp. 1-5, 2006.

[8] Rondineli Rodrigues Pereira, Carlos Henrique da Silva, Luiz Eduardo Borges da Silva,et al,New Strategies for Application of Adaptive Filters in Active Power Filters,IEEE Transactions on Industry Applications, 47(3), pp. 1136-1141, 2011.

[9] ZENG Ji-yong,DING Hong-fa,DUAN Xian-zhong,A Harmonics Detection Method Based on Mathematical Morphology,Automation of Electric Power Systems, 29(6), pp.55-59, 2005.

[10] WANG Jing, LIU Di-chen, LIU Pan,et al,A New Algorithm for Random Harmonic Current Detection Based on Mathematical Morphology, 2009 Global Congress on Intelligent Systems, IEEE: Xiamen, pp. 365-369, 2009.

[11] Ou-yang Hua, Bu Le-ping, Yang Zhong-lin,Voltage Sag Detection Based On Dq Transform and Mathematical Morphology Filter, Procedia Engineering,23, pp. 775-779, 2011.

[12] Yingjie He, Yunping ZOU, Jian Tang,et al, Digital Realization of a Novel Detection Algorithm Based on Instantaneous Reactive Power Theory, The 33rd Annual Conference ofthe IEEE Industrial Electronics Society (IECON), IEEE: Taipei,Taiwan, pp. 1869-1874, 2007. 